\title{
A Contour-Based Segmentation Algorithm for Triangle Meshes in 3D Space
}

\begin{abstract}
This paper introduces the first contour-based mesh segmentation algorithm that we may find in the literature, which is inspired in the edge-based segmentation techniques used in image analysis, as opposite to region-based segmentation techniques. Its leading idea is to firstly find the contour of each region, and then to identify and collect all of its inner triangles. The encountered mesh regions correspond to ups and downs, which do not need to be strictly convex nor strictly concave, respectively. These regions, called relaxedly convex regions (or saliences) and relaxedly concave regions (or recesses), produce segmentations that are less-sensitive to noise and, at the same time, are more intuitive from the human point of view; hence it is called human perception-oriented (HPO) segmentation. Besides, and unlike the current state-of-the-art in mesh segmentation, the existence of these relaxed regions makes the algorithm suited to both nonfreeform and freeform objects.
\end{abstract}

Keywords: Mesh segmentation, relaxed convexity, human perception-oriented segmentation

\section{Introduction}

Mesh segmentation is a fundamental procedure in areas as diverse as geometric modeling, computer-aided design, computer graphics, and so forth [37]. This procedure consists in partitioning a mesh into a number of sub-meshes in conformity with some convexity criterion. Intuitively, this is equivalent to detect and delimit the ups (saliences) and downs (recesses) of the mesh, as illustrated in Fig. 1. But, as argued by Attene et al. [3], mesh segmentation can be driven by either geometric criteria or semantic criteria or both.

In geometry-based segmentation techniques, the mesh is divided into a number of sub-meshes or regions that satisfy some geometric property (e.g., curvature, distance to a fitting plane, etc.). On the other hand, in semantics-based segmentation techniques, the division of a mesh into sub-meshes takes place when each sub-mesh delimits a perceptually meaningful region (e.g., an arm of the human body). In this respect, Biederman [6] states that the people perceive objects as collections of parts, while Hoffman [20] refers that the human vision defines boundaries of parts along the negative minima of principal curvatures. Note that, in a way, Hoffman's assertion implies that the meaningful parts are essentially convex.

Hoffman's assertion has inspired the development of our algorithm, so we follow the leading idea of how human vision perceives the boundaries of parts. Let us say that our algorithm has been also inspired in the well-known family of edge-based segmentation algorithms found in image analysis and algorithms. With these edge-based algorithms, we first find the boundaries of each region, filling it afterwards; hence, the contour-based segmentation (region contour or boundary first, then its interior). On the contrary, the region-based algorithms work in the other way round: first the region is formed incrementally, being its frontier defined by some stopping condition.

As far as we know, we are here proposing the first contourbased segmentation in the domain of mesh segmentation in 3D.
For this purpose, the point membership test (PMT) is here used as a convexity classifier, i.e., we use PMT to classify edges as convex, concave or flat. PMT is a particular case of the SMC (set membership classification) test, which is very popular in CSG (constructive solid geometry) modeling, and has been around for the last three to four decades [43]. Nevertheless, recesses and saliences on the mesh are not classified in a so strict manner in terms of convexity. We introduce the notion of relaxed convexity to classify those shape features of the mesh. A salience is a relaxedly convex region, while a recess is a relaxedly concave region. A relaxedly convex region is not strictly convex, i.e., it admits small concavities. On the other hand, a relaxedly concave region is not strictly concave, i.e., it admits small convexities. This shape relaxation allows us to minimize the effects of noise related to over-segmentation, and makes it suited to the segmentation of freeform objects such as, for example, the ant shown in Fig. 1.

The remainder of the paper is organized as follows. Section 2 briefly reviews the related work existing in the literature. Section 3 details the background that is on the basis of our algorithm, including PMT and the concept of relaxed convexity. Section 4 outlines our contour-based segmentation algorithm, called HPO segmentation. Section 5 details the region filling step of HPO segmentation algorithm. Section 6 introduces the mesh smoothing step (a Laplacian filter, in particular) for noisy meshes, which uses the cumulative area histogram analysis to identify whether a mesh is noisy or not. Section 7 describes the region merging step of HPO segmentation algorithm. Section 8 presents the most relevant experimental results produced by the HPO segmentation algorithm, in particular the benchmarking results produced by Princeton's benchmark software. Finally, Section 9 concludes the paper. 


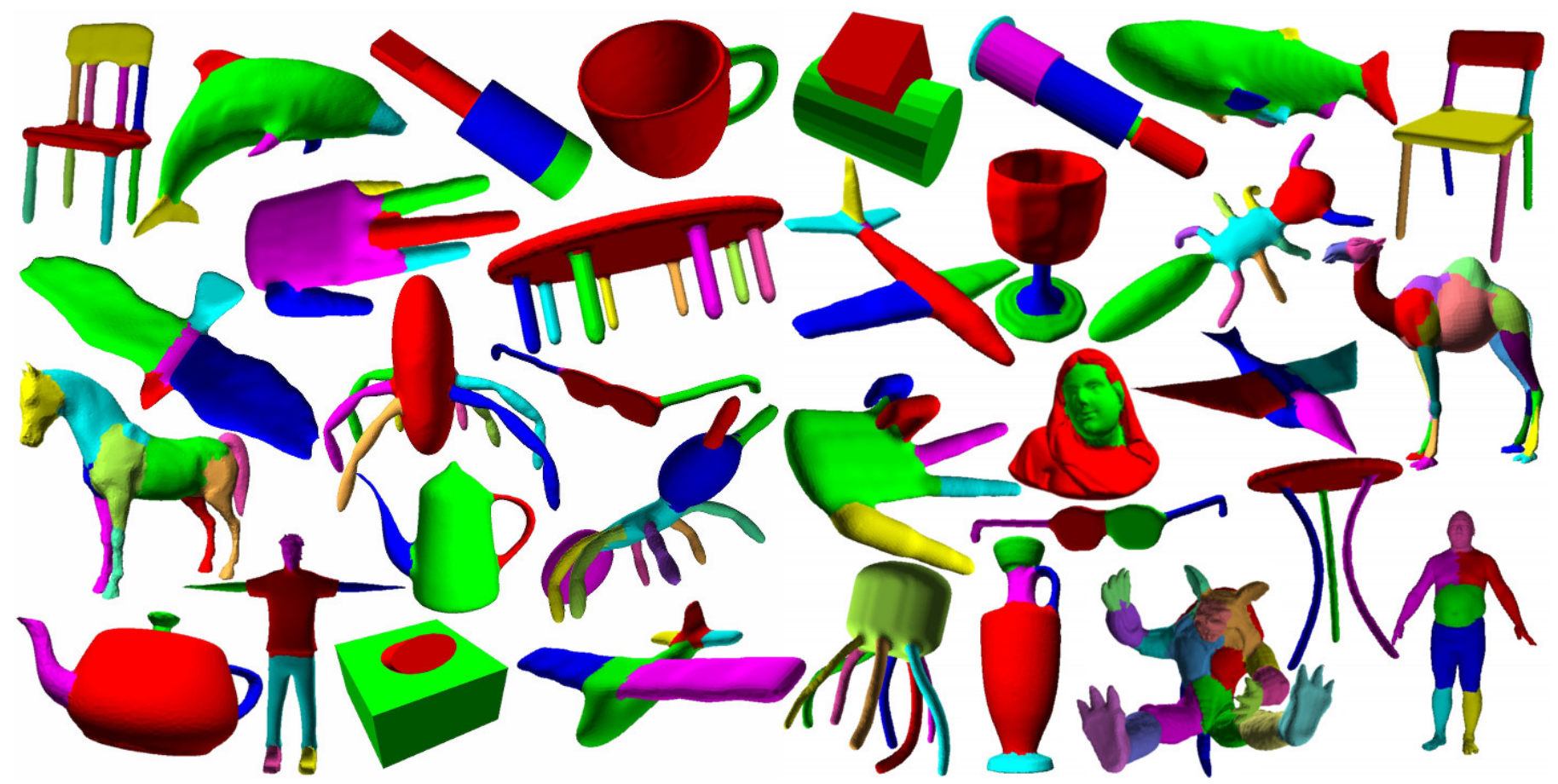

Figure 1: Segmented meshes (or models) using the human perception-oriented (HPO) segmentation.

\section{Related Work}

Intuitively, mesh segmentation consists in dividing a mesh into meaningful sub-meshes (or regions). With reference to the structural dimension of mesh regions, segmentation algorithms can be categorized as follows: volume-based, surface-based, and skeleton-based. These three segmentation categories are all based on geometry, including concepts of the convexity theory, topology, homology, etc. Independently of the nature of the segmentation technique, most algorithms make usage of some geometric or topological criteria that guide the segmentation of a given mesh [37]. Examples of geometric criteria are the curvature [24], geodesic distances [46], dihedral angles [48], shape diameter function [38], planarity [21], symmetry [34], convexity [27] [31], concavity [25] [15], volume [19], etc. Topological criteria include Reeb graphs [42] and spectral analysis [47].

Volume-based segmentation (also called part-type segmentation) algorithms can be considered as the former category of segmentation algorithms. In this case, the segments are volumes. This family of algorithms follows the principle of decomposing a $3 \mathrm{D}$ volumetric object into a set of convex subobjects (or convex volumes). The basic problem of convex decomposition of polyhedra was firstly addressed by Chazelle [11] [5] [9], but such decompositions usually are costly in terms of time performance and memory space. More amenable decompositions, called approximate convex decompositions (ACD), have been proposed in the literature [29] [26] [15]. This sort of decomposition is based on a measure of concavity; for example, the ACD proposed in [30] is guided by the volume ratio between the actual object and its convex hull, Ghosh et al. [15] propose a new strategy that improve the results of the ACD, while the one due to Kraevoy et al. [26] is generated by measuring the average distance from all object's triangles to the object's convex hull.

In surface-based segmentation (also known as surface-type) algorithms, the segments are regions of a 2D triangle mesh. Each region consists of a number of connected triangles that have similar geometric properties (e.g., convexity, curvature, etc.). In the literature, we find the following major sub-categories surface-based algorithms: region growing [10] [21], watersheds [33] [48], hierarchical clustering [14], iterative clustering [36], [23], spectral clustering [32], [35], and fuzzy clustering [23].

In skeleton-based segmentation (also known as skeletonization) algorithms, the segments are line segments. The input is either a 3D volumetric mesh or a 2D surface mesh, but the output is a 1D skeleton that represents the structural shape of the mesh. A skeleton provides us with the one-dimensional topological shape of a given higher-dimensional object. Generating such a skeleton is a process known as skeleton extraction or skeletonization [8] [23]. Examples of automatic skeletonization techniques are found in the literature, including the Medial Axis Transform (MAT) [7], Shock graph [41] (i.e., another MAT representation), and Reeb graph extracted from various Morse functions [39].

Note that our algorithm falls into the category of surfacebased algorithms, but it does not fit any of its sub-categories. Making a parallel with the two more important families of segmentation algorithms we may find in image analysis and processing [17], a region-based segmentation algorithm starts somewhere in the interior of each region and stops on its frontier, 


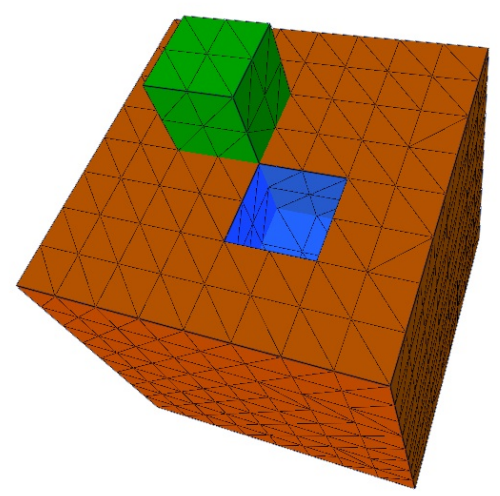

(a)

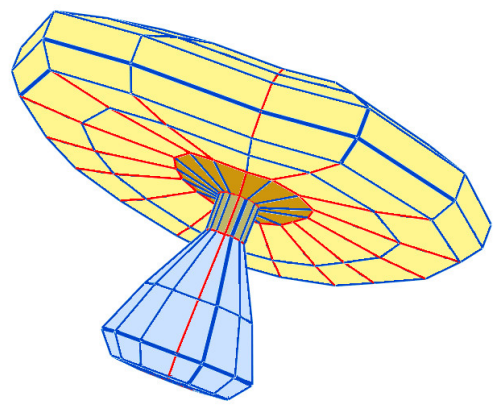

(b)

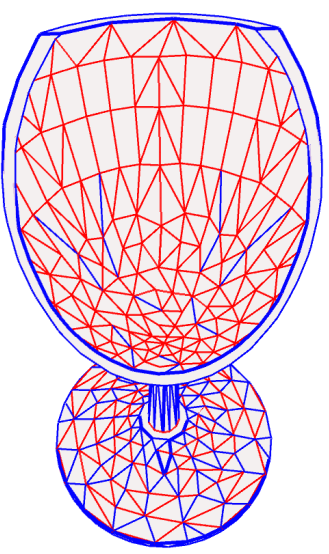

(c)

Figure 2: Three different meshes: (a) a mesh with two saliences (a brown salience and a green salience) and a blue recess; (b) a mushroom with four segments or regions (convex edges in blue and concave edges in red); (c) a calyx with a cut (convex edges in blue and concave edges in red).

while a contour-based segmentation algorithm starts on the frontier of each region and stops in its interior. This means that, we do not need to calculate neither maxima nor minima to start with the segmentation, nor to use other suited, sophisticated mechanisms to determine the curvature over the mesh as those we find commonly nowadays.

\section{Theoretical Background}

According to Hoffman's assertion mentioned in Section 1, the human vision delineates the boundaries of object's regions along the negative minima of principal curvatures, what implies that meaningful regions of an object are convex. This agrees with the fact that there is only lack of matter (or existence of recesses) when the matter exists (or existence of saliences). But, when we talk about the convex regions, in fact, in most cases, we are talking about regions that we call relaxedly convex regions. This is illustrated in Fig. 2(b), where the four meaningful parts of the mushroom are not strictly convex, i.e., we tend to observe the convexity in large. For example, the mushroom cap (i.e., the top region) is a relaxedly convex region since it mostly consists of convex edges in blue, although it also possesses concave edges in red.

\subsection{Edge Classification}

Evaluating the convexity of an edge bounding a mesh triangle can be reduced to the problem of classifying a point in relation to a plane. Let us then use the vectorial equation of the plane $\alpha$ given by the dot product $\vec{N} \cdot \overrightarrow{Q P}=0$, where $\vec{N}$ is the normal vector of the triangle at one of its points $Q$ and $\overrightarrow{Q P}$ stands for a vector in $\alpha$ defined by $Q$ and any other point $P$ belonging to $\alpha$. Assuming that $N=(a, b, c), Q=\left(x_{0}, y_{0}, z_{0}\right)$ and $P=$ $(x, y, z)$, we easily obtain the Cartesian equation of the plane as $\alpha(x, y, z): a x+b y+c z+d=0$, where $d=\left(-a x_{0}-b y_{0}-c z_{0}\right)$.

It is clear that the plane $\vec{N} \cdot \overrightarrow{Q P}=0$ divides $\mathbb{R}^{3}$ in two halfspaces, i.e., the positive half-space $\vec{N} \cdot \overrightarrow{Q P}>0$ and the negative half-space $\vec{N} \cdot \overrightarrow{Q P}<0$. The normal $\vec{N}$ points to the positive half-space (i.e., outside), so that we can easily test whether an edge is convex or not using the plane of the first of its incident triangles and the opposite vertex (i.e., the third vertex) of the second triangle.

Let $\vec{N}_{1}$ and $\vec{N}_{2}$ be the normal vectors of the first and second triangles incident on a given edge, respectively. Also, let $P_{1}$ and $P_{2}$ be the opposite vertices of the first and second triangles. Thus, after calculating $\Delta=\overrightarrow{N_{1}} \cdot \overrightarrow{P_{1} P_{2}}$, we have:

- If $\Delta=0$, then the triangles are coplanar, and the edge is said to be flat;

- If $\Delta<0$, the second triangle lies in the negative side of the plane $\alpha$, that is, the edge shared by those two triangles is said to be convex;

- If $\Delta>0$, the second triangle lies in the positive side of $\alpha$, that is, the edge shared by those two triangles is said to be concave.

Note that this criterion, here called PMT, can be used to know whether or not an edge is convex without computing the dihedral angle between its incident triangles. Besides, the PMT does not suffer from the ambiguity of the dihedral angle in evaluating the convexity of edges. During testing, we observed that PMT criterion is more than fifty percent faster than the dihedral angle criterion in the evaluation of the convexity of edges.

\subsection{Triangle Classification}

The PMT is a strict convexity criterion for edges. An edge shared by two triangles is flat if $\Delta=0$, convex if $\Delta<0$, and concave if $\Delta>0$. Considering that we have three different types of edges and also that a triangle has tree bounding edges, we end up having $3^{3}=27$ types of triangles classified in terms of their edges. Nevertheless, after eliminating the redundant types of triangles, we end with 10 different types of triangles, which are shown in Fig. 3. 


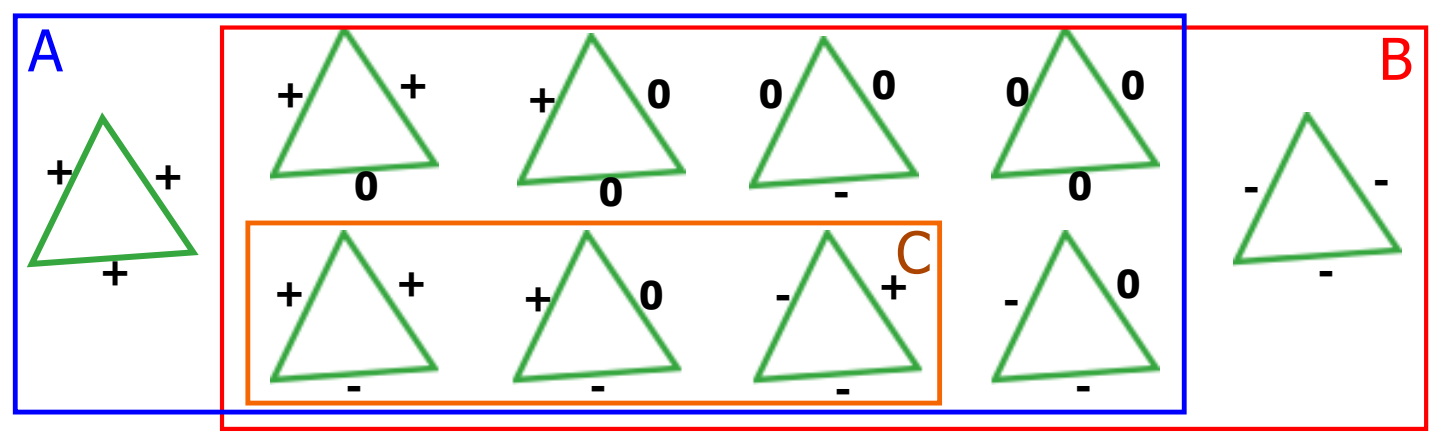

Figure 3: Edge types of a triangle: (+) for convex edges; (0) for flat edges; and (-) for concave edges. $A$ stands for the set of triangles used for build relaxedly convex regions; $B$ stands for the set of triangles used for build relaxedly concave regions; and $C$ stands for the set of boundary triangles.

Definition 1. A triangle falls into one of the following categories:

1. A triangle is of the type +3 convex (or simply convex) if its edges are all convex (e.g., the triangle on the left hand side in Fig. 3);

2. A triangle is of the type +2 convex if it has two convex edges and one flat edge;

3. A triangle is of the type+1 convex if it has one convex edge and two flat edges;

4. A triangle is flat if its edges are all flat;

5. A triangle is of the type-3 concave (or simply concave) if its edges are all concave (e.g., the triangle on the right hand side in Fig. 3);

6. A triangle is of the type-2 concave if it has two concave edges and one flat edge;

7. A triangle is of the type-1 concave if it has one concave edge and two flat edges;

8. A triangle is of the type+2-1 undefined if it has two convex edges and one concave edge;

9. A triangle is of the type+1-2 undefined if it has one convex edge and two concave edges;

10. A triangle is of the type+1-1 undefined if it has one convex edge, one concave edge, and one flat edge.

In short, we have tree types of convex triangles, one type of flat triangles, three types of concave triangles, and three types of undefined triangles in terms of convexity.

\subsection{Region Classification}

Let us now to come up with definitions about regions, which are sustained on the definitions introduced in previous sections.

Definition 2. A region of a triangle mesh is a connected set of triangles (or sub-mesh) that locally has the topological type of a 2-dimensional disc.

Definition 3. A region is said to be convex if the following conditions are satisfied:

(1) (Interior Condition). Its triangles belong to the boundary of its convex hull;
(2) (Boundary Condition). It is delimited by at least one cycle of concave edges.

A consequence of the first condition is that the inner triangles of a convex region are convex (any type) or flat. It also follows that triangles with concave edges are not admissible for inner triangles of a convex region. Additionally, those two conditions imply that each outer triangle of a convex region has a single concave edge, that is, each outer triangle belongs to one the following types: type- 1 concave, type $+2-1$ undefined, or type+1-1 undefined. For example, the outer triangles of the salience (in green) shown in Fig. 2(a) fall into two types: type1 concave and type+1-1 undefined. Note that a convex region may be bounded by two cycles of concave edges, as it is the case of the smallest region (in green) of the five-region shaft depicted in Fig. 1.

Analogously, a concave region also satisfies the interior condition (1) above. By contrast, the boundary of concave region consists of at least one cycle of convex edges. For example, the depression (in blue) shown in Fig. 2(a) is a concave region that is bounded by a cycle of convex edges. Thus, the inner triangles of a concave region are concave (any type) or flat, while those of a convex region are convex (any type) or flat.

In respect to relaxedly convex regions, we can say the following:

Definition 4. A region is said to be relaxedly convex if the following condition is satisfied:

(1) (Relaxed Interior Condition). Its triangles do not necessarily belong to the boundary of its convex hull;

(2) (Boundary Condition). It is delimited by at least one cycle of concave edges.

That is, a relaxedly convex region does not satisfy the interior condition of a convex region; however, convex regions and relaxedly convex region share the same boundary condition. This means that we may have concave edges in the interior of a relaxedly convex region since that they do not form a loop. For example, the mushroom cap shown in Fig. 2(b) is a relaxedly convex region that has concave edges (in red) in its interior; in addition, the region adjacent to the mushroom cap 


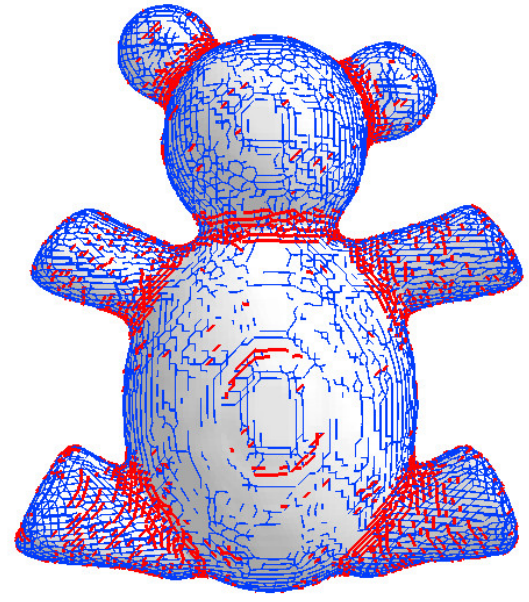

(a)

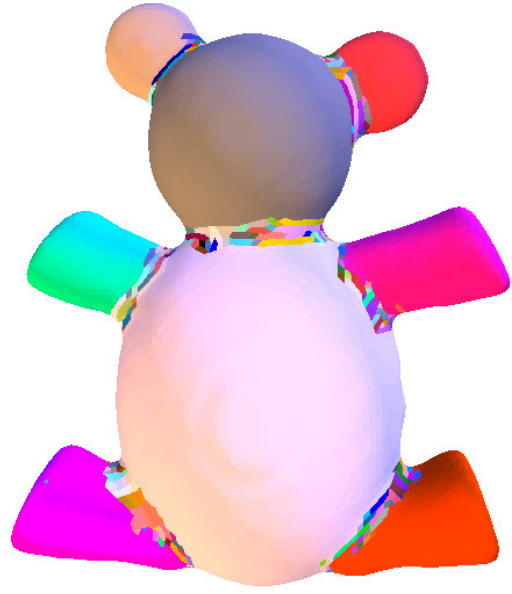

(b)

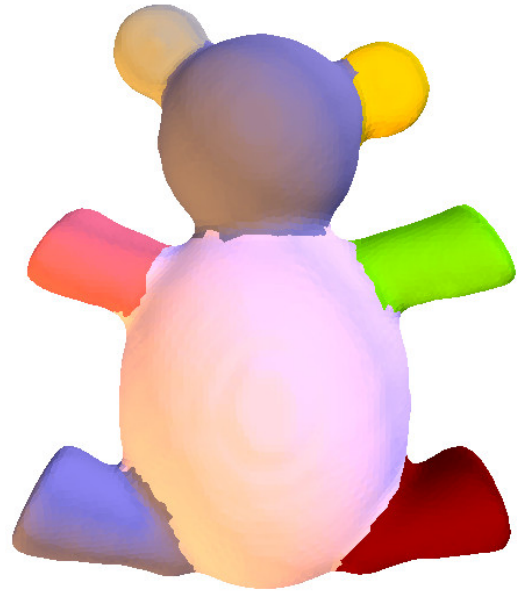

(c)

Figure 4: Algorithm steps for a teddy mesh: (a) after edge classification (convex edges in blue and concave edges in red) and triangle classification; (b) after region filling; (c) after region merging.

shown in Fig. 2(b) is a relaxedly convex region with two bounding cycles of concave edges.

Similar to relaxedly convex regions, a relaxedly concave region also satisfies the relaxed interior condition. That is, we may find convex edges in the interior of a relaxedly concave region; however, they cannot form a cycle. On the other hand, concave regions and relaxedly concave regions share the same boundary condition, that is, they are delimited by one or more cycles of convex edges. For example, the inside of the calyx shown in Fig. 2(c) is a relaxedly concave region that has convex edges (in blue) in its interior.

It is worthy noting that a convex region is also relaxedly convex, but not vice-versa; similarly, a concave region is also relaxedly concave, but not vice-versa. Relaxed convexity is particularly important in the segmentation of freeform objects; for example, the legs of the octopus depicted in Fig. 1 are examples of relaxedly convex regions. But, it is also suitable for feature recognition of artifacts as those produced by geometric kernels and CAD systems (e.g., the two mechanical shafts in the Fig. 1). In short, unlike other segmentation techniques that divide a mesh into convex, concave and saddle regions, ours divides a mesh into relaxedly convex regions (saliences) and relaxedly concave regions (recesses), preventing so the oversegmentation of the mesh as much a possible.

\section{Contour-Based Segmentation Algorithm: Overview}

Considering the previous theoretical elements on convexity, the steps of our algorithm are as follows:

1. Edge Classification. Classify each edge of the mesh as either convex edge, concave edge or flat edge. This classification is performed using the point membership test (PMT) instead the dihedral angle. See Section 3.1 for further details.
2. Triangle Classification. This procedure builds on the edge classification above, as formalized by Definition 1 in Section 3.2.

3. Region Filling. Form mesh regions from boundary triangles, that is, triangles having at least one edge in the boundary of each region. However, the algorithm is flexible enough to start with any triangle to form a region. See Section 5 for more details.

4. Mesh Smoothing. This step starts by checking whether or not a given mesh is noisy (e.g., Armadillo's mesh shown in Fig. 8(a)) or not. Any noisy mesh is subject to a smoothing procedure, after which such mesh is re-segmented repeatedly while the noise persists above a given threshold. Non-noisy meshes are not subject to any smoothing procedure, i.e., the mesh is immediately dispatched to the region merging step.

5. Region Merging. Merge adjacent regions if appropriate. See Section 7 for further details.

The main steps of the algorithm are illustrated in Fig. 4. In Fig. 4(a), we have the edge classification (convex edges in blue, concave edges in red, and flat edges in grey), while all the triangles are drawn in grey. Fig. 4(b) illustrates the region filling step. The inwards expansion (i.e., from the boundary to interior) of each region stops when there is not more boundary and interior triangles to fill in. Finally, Fig. 4(c) shows us the teddy after the region merging step.

Our algorithm produces a kind of human-meaningful segmentation (i.e., HPO segmentation) in a way that saliences (relaxedly convex regions) are formed before recesses (relaxedly concave regions). As illustrated in Fig. 5(a), the saliences must be found before recesses because the resulting mesh segmentation approximately agrees with the way humans perceive the shape of 3D geometric objects. In fact, the ground-truth segmentations produced by the Princeton benchmark [13] show us that humans essentially have an additive shape perception of $3 \mathrm{D}$ 


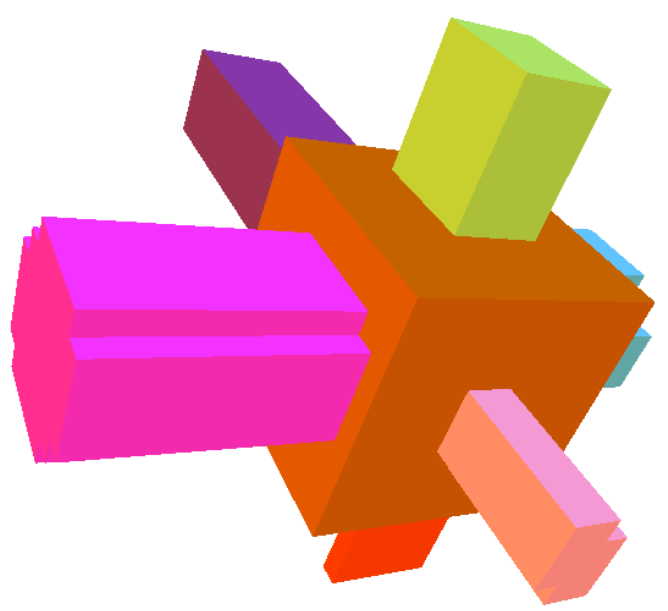

(a)

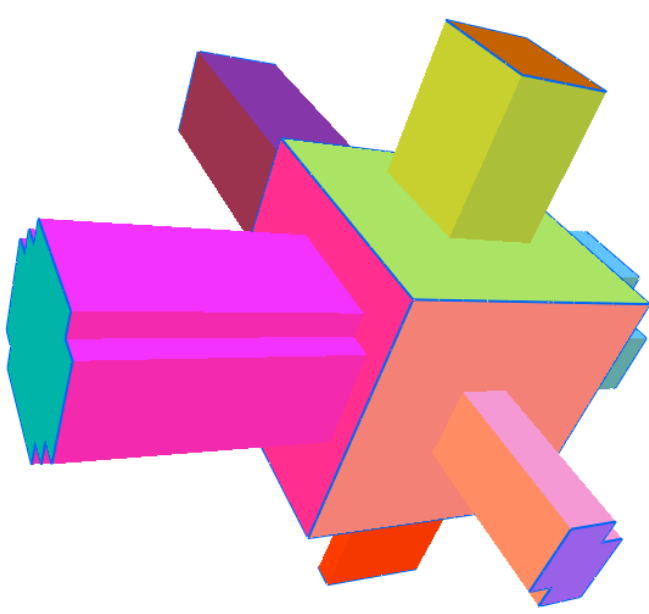

(b)

Figure 5: HPO segmentation: (a) first convex regions, then concave regions; (b) first concave regions, then convex regions.

objects in the sense that each object is mainly seen as a union of saliences. To reinforce this idea, let us consider that the priority of segmentation goes to recesses at detriment of saliences. As shown in Fig. 5(b), the resulting segmentation endows a subtractive perception of shape to $3 \mathrm{D}$ objects, which is not so natural from the human point of view.

\section{Region Filling}

Let us assume that all the mesh edges have been labeled in terms of their convexity: either convex, or concave, or flat. Also, the mesh triangles have been labelled in conformity with Definition 1. Then, we can proceed to filling in relaxedly convex regions in first place, and relaxedly concave regions afterwards.

\subsection{Filling Relaxedly Convex Regions}

Filling in a relaxedly convex region starts with one of its boundary triangles, i.e., with one undefined triangle (cf. undefined triangles of the set $\mathrm{C}$ in Fig. 3). Recall that a boundary triangle has at least a convex edge and a concave edge. The filling procedure follows the door-in-door-out principle [1]. An edge that bounds a triangle acts as door to get in or get out such a triangle, or still to stop the local expansion of a region. Given a relaxedly convex region, we consider that convex edges (and also flat edges) in the frontier of a triangle are open doors to neighbor triangles, so that such a region spreads inwards. In other words, if a door-in edge of a triangle is convex, its doorout edges must be convex or flat; in this case, a concave edge is a boundary edge that blocks the expansion of such a relaxedly convex region.

The procedure to form relaxedly convex regions (or saliences) of a mesh is described in Algorithm 1. As noted above, filling in a relaxedly convex region starts with a boundary triangle (cf. lines 4 to 12 in Algorithm 1). For this purpose, and according to the door-in-door-out principle, one examines whether or not the neighbor triangles may be added to the relaxedly convex region (cf. line 7), being then the growing process iterated on each previously added triangle. Recall that the filling of a relaxedly convex region is barred by concave edges (i.e., its frontier), as expressed by the condition of the while loop in line 7.

It is worthy noting that Algorithm 1 applies to all boundary triangles, i.e., no boundary triangle is left to the posterior formation of relaxedly concave regions. This means that Algorithm 1 may produce small relaxedly convex regions consisting of two triangles; for example, the isolated convex edge (in blue) in the interior of the calyx in Fig. 2 may originate a relaxedly convex region consisting of its two incident triangles. As described in Section 7, these small regions will be later absorbed by larger regions in their vicinity.

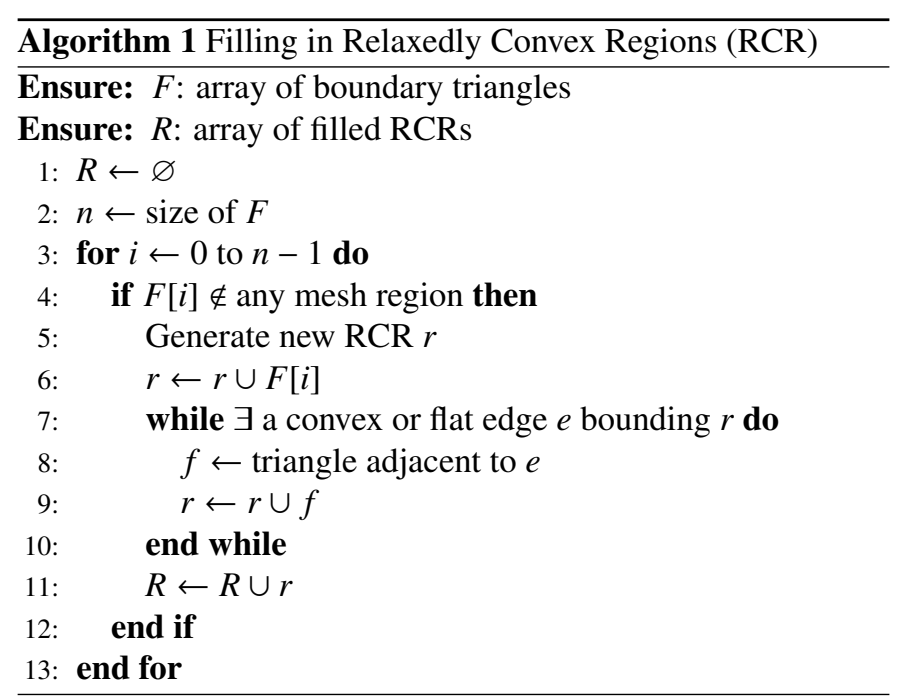


II Armadillo || Teddy

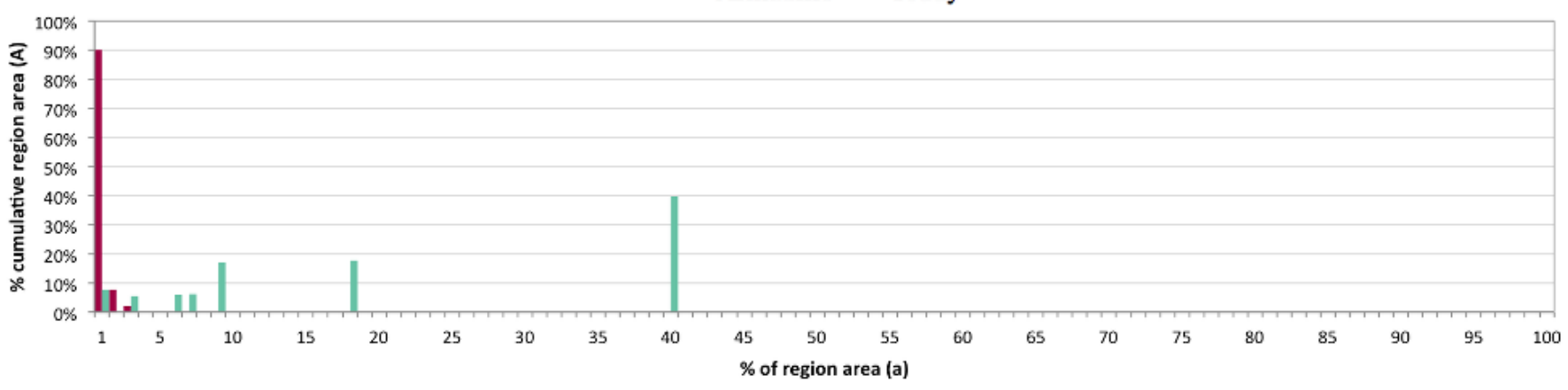

Figure 6: Cumulative area histogram of Armadillo and Teddy meshes (models 161 and 281 models of Princeton Benchmark).

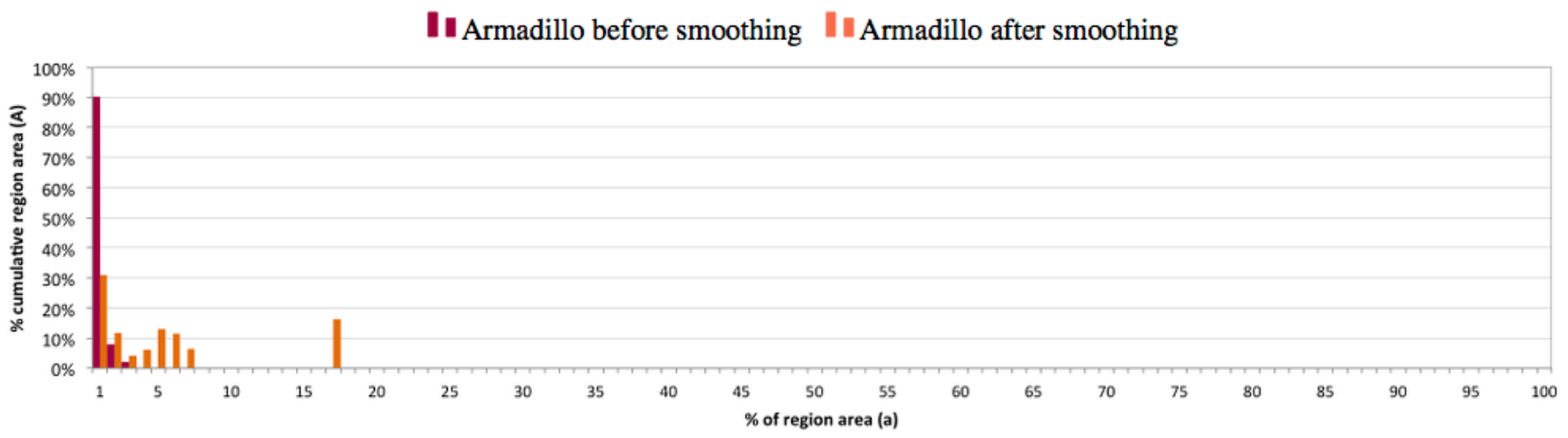

Figure 7: Cumulative area histogram of Armadillo before and after smoothing (model 281 of Princeton Benchmark).

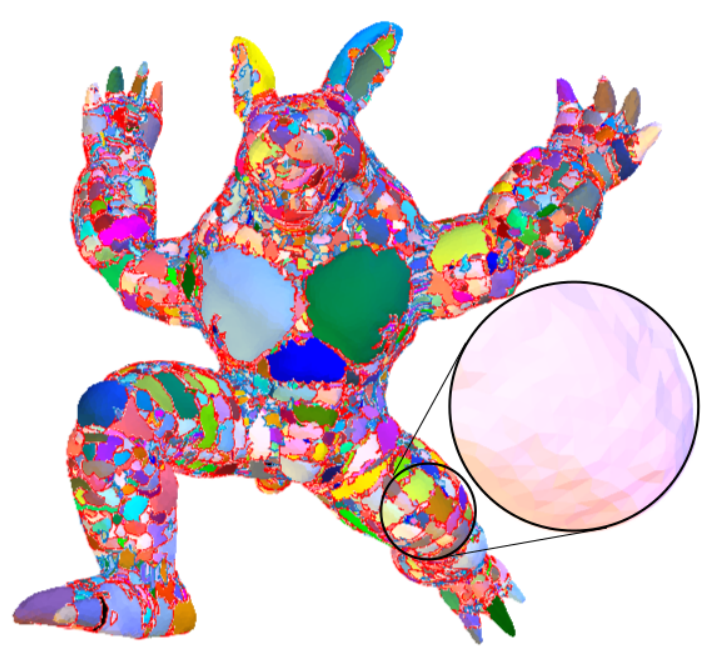

(a)

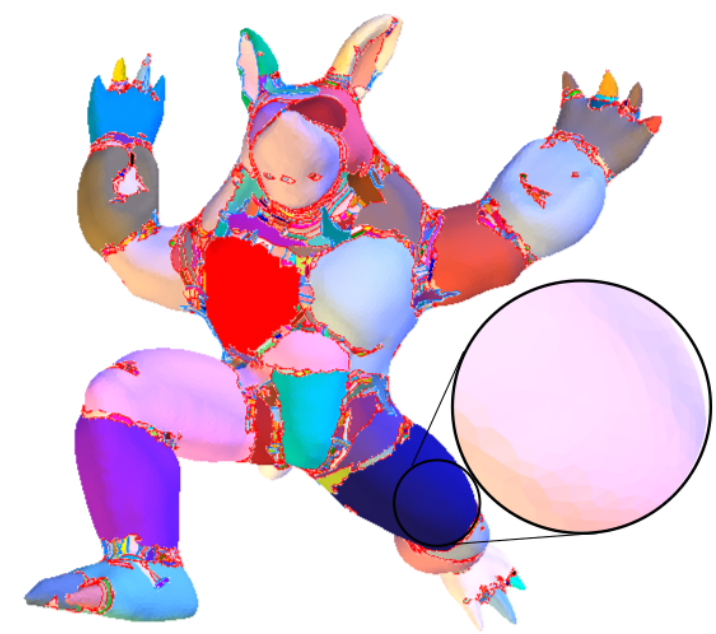

(b)

Figure 8: Armadillo's mesh after the region filling step (model 281 of Princeton benchmark): (a) original mesh; (b) smoothed mesh.

\subsection{Filling Relaxedly Concave Regions}

Likewise, the procedure to form relaxedly concave regions (or recesses) is done by exchanging the roles of convex and concave edges in Algorithm 1. Besides, the algorithm to filling in relaxedly concave regions does not iterate on the array of boundary triangles, but on the array of concave triangles (i.e., type- $k$ concave triangles, with $k=1,2,3$ ).

It is clear that if the priority of the segmentation had been given to recesses at detriment of saliences, the algorithm would iterate on the array of boundary triangles, while saliences would be formed from the array of convex triangles (i.e., type- $k$ convex triangles, with $k=1,2,3)$. But, as shown in Fig. 5(b), the resulting segmentation in this case would not be so close to the human perception about shape of 3D objects.

Remarkably, the region filling step suffices to correctly segment meshes of nonfreeform objects (e.g., mechanical parts). 
Even some freeform objets do not need the supplementary step of region absorbing to attain their final segmentations, as it is the case of the mushroom shown in Fig. 2. This makes our algorithm particularly adequate for feature recognition of artifacts as those produced by geometric kernels and CAD systems (e.g., the two mechanical shafts in Fig. 1).

\section{Mesh Smoothing}

It is widely known that noise may undermine the segmentation of a given mesh. We follow the notion of noise in image processing and analysis [17], so that high frequencies of a signal (or image) correspond to rapid oscillations of a mesh. Intuitively, this means that a noisy mesh possesses a large number of small segments, i.e., it is over-segmented.

In order to cope with noisy meshes, we need to check beforehand whether a given segmented mesh is noisy or not; for this purpose, we use histogram analysis. Identified a noisy mesh, we apply a Laplacian filter to reduce its noise.

\subsection{Area Histogram Analysis}

By analysing the cumulative relative area histogram of a given segmented mesh, we are able to decide whether such a mesh is noisy or not. For example, Fig. 6 shows the cumulative histogram of relative region areas of two meshes, Teddy's mesh (Fig. 4) and Armadilo's mesh (Fig. 8), where $a$ stands for the region area percentage in relation to the total mesh area, also called relative region area, while $A$ denotes the cumulative region area percentage in relation to the total mesh area, here called cumulative relative region area.

Intuitively, we see that Teddy's mesh is not much noisy because the cumulative relative area of its smaller regions (i.e., regions with less than 1 percent of relative area) is small (i.e., less than 10 percent); also, we have 1 region (the body trunk) with $a=A=40 \%, 1$ region (the head) with $a=A=18 \%, 2$ regions (the legs) with $a=9 \%$ and $A=18$, and so on. On the contrary, we observe that Armadillo's mesh is noisy because $90 \%$ of the total area of the mesh is made up of the smaller regions, i.e., regions with less $1 \%$ of relative area. Note that these smaller regions add up to the first bin (on the left hand side) of the histogram, here called noise bin.

More specifically, we assume that a mesh is noisy if the percentage of the cumulative region area $(A)$ of the noise bin $[0,1$ [ is at least twice greater than the one of the second highestranked bin. This noise-based criterion is empirical and was obtained after an exhaustive number of experiments.

\subsection{Laplacian Smoothing}

We use Laplacian smoothing to reduce the noise of a given noisy mesh. The Laplacian operator replaces the position of each vertex by the mean average of the positions of its adjacent vertices [18] [2] [44]. Therefore, the Laplacian operator is a local shape operator. It works as a low-pass filter, i.e., it preserves the large mesh oscillations (corresponding to low frequencies of a signal) and throws away the small mesh oscillations (corresponding to high frequencies of a signal).
The Laplacian operator is applied to the mesh as many times as necessary, i.e., while the noise-based criterion is satisfied. For example, the Armadillo's histogram (in orange) in Fig. 7 was obtained after four iterations of the Laplacian operator. It is worth noting that after applying the Laplacian operator to the mesh, the flow control of the segmentation algorithm goes back to its first step, in order to proceed to the re-segmentation of the mesh. For example, the Armadillo's segmentation shown in Fig. 8(b) is the result of applying the Laplacian smoothing twice. Once the noise-based criterion is no longer satisfied, the mesh vertices recover their original positions before proceeding to the region merging step. It should be also noted that the smoothing step described in the present section does not apply to the majority of the meshes; it only applies to noisy meshes.

\section{Region Merging}

The region merging step is essentially necessary for freeform objects (e.g., human beings and other animals). These freeform objects are the most challenging objects in the segmentation process of our algorithm because they feature many variations in curvature, what provokes the over-segmentation of the mesh. In large extent, the ups and downs of the freeform objects led us to come up to the notion of relaxed geometry, which is at the core of our algorithm. Thus, the region merging aims at further reducing the over-segmentation of the mesh.

In the region merging step, we decided to use the algorithm proposed by Chen and Georganas [12]. The leading idea of this algorithm is to reduce the number of small regions (or segments) by merging of these small regions with adjacent larger regions. The main steps of the merging procedure are presented in Algorithm 2.

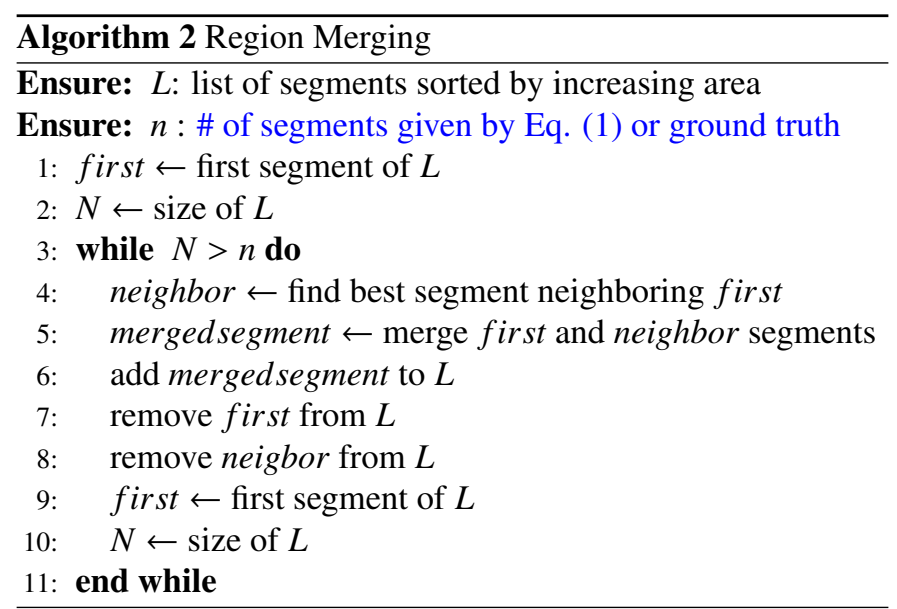

The algorithm requires a preliminary sorting of the list of regions in terms of increasing areas. Basically, a small region is merged with its adjacent region with which it shares the longest path of its boundary (cf. line 4 of Algorithm 2). The stopping condition (cf. line 3 of Algorithm 2) is satisfied when the number of regions of the segmentation equals the value given by

$$
n=\sum_{i=2}^{100} \frac{A_{i}}{a_{i}}
$$


where $a_{i}$ stands for the relative region area associated to each bin of the histogram, and $A_{i}$ denotes the corresponding cumulative region area. Note that the index $i$ of the first bin of the histogram (i.e., $i=1$ that corresponds to the interval [0,1[ in the horizontal axis) is not considered in Eq. (1) because it accumulates areas of noisy regions.

Eq. (1) provides a number of segments that is similar to the one of the ground truth defined by the Princeton benchmarking [13]. Nevertheless, as suggested by Chen et al. [13], we also used the value of $n$ of the ground truth in order to not give advantage to any algorithm involved in the comparison undertaken in Section 8. Morever, only 2 out of 7 benchmark algorithms are able to calculate the number of segments automatically.

\section{Experimental Results}

\subsection{Testing Setup}

The HPO segmentation algorithm was designed and implemented on an Intel Core Duo 2.4 computer running a Mac OS $\mathrm{X}$ operating system, and using the OpenGL User Interface Library (GLUI), which is a $\mathrm{C}++$ user interface library based on the OpenGL Utility Toolkit (GLUT).

\subsection{Benchmarking}

We have used the Princeton benchmarking to compare our algorithm to the state-of-the art of algorithms in mesh segmentation [13]. This benchmarking tool provides 19 categories of models, with each category comprising 20 shapes (i.e. 380 models). This tool also supplies the ground truth of 4300 humangenerated segmentations, being provided on average 11 humangenerated segmentations concerning distinct poses of each shape.

The Princeton segmentation benchmarking provides quantitative comparisons of human-generated segmentations (i.e., ground truth) and computer-generated segmentations produced by the following seven algorithms: k-means [40], random walks [28], fitting primitives [4], normalized cuts [16] randomized cuts [16], core extraction [22] and shape diameter function [38]. It is worth noting that the benchmarking software does not include the codes of those seven algorithms, but only segmentations produced by them, which work as input data for the benchmarking software. Therefore, before running the benchmarking itself for the eight algorithms (including ours), we added the segmentations generated by our algorithm to the benchmark.

In order to compare the segmentations produced by those eight algorithms (including our algorithm), the Princeton benchmarking tool uses the following four metrics: Rand Index (RI), Cut Discrepancy (CD), Consistency Error (CE), and Hamming Distances (HD). The CD metric focuses on boundary errors, while the other three metrics focus on region dissimilarities. Fig. 9 compares our algorithm to the seven segmentation algorithms of the benchmark, with reference to the aforementioned four benchmark metrics. Note that the metrics are consistent with one another, in the sense that segmentation algorithms have the same relative performance with regard to such metrics.
A more detailed comparison is presented in Table 1, whose results show that on average the HPO algorithm ranks first for the four benchmarking metrics.

\subsection{Discussion and Limitations}

In a previous version of the HPO algorithm, the smoothing step was not part of the algorithm. Even so, the HPO algorithm was ranked first for the RI, CD, and HD metrics, while it ranked second for the CE metrics. In this latter metric, the RandCuts algorithm performed better than our algorithm, but not that much. This happens because CE metrics provide better results when the number of regions is higher than the humangenerated regions of the ground truth, as it is the case of the RandCuts algorithm. In fact, according to Chen et al. [13], the $\mathrm{CE}$ metrics have the "problem that they normally provide better scores when two models have different numbers of segmentations". Consequently, we can fit small regions produced by RandCuts in bigger human-generated regions. By contrast, the HPO algorithm tends to produce the same number of regions as in the ground truth, so it becomes more difficult to fit a HPO region in a ground truth region. This drawback of HPO algorithm in respect to $\mathrm{CE}$ metrics was solved using the additional step of mesh smoothing for noisy meshes, as it is the case of Armadillo's mesh shown in Fig. 8. In this way, the HPO algorithm ends up being more robust in respect to mesh noise.

Although the mesh noise is a problem for many algorithms, it is not less true that the absence of mesh noise may be also a problem for a proper segmentation of a given mesh. For example, the HPO algorithm cannot produce an adequate segmentation for a T-shape provided that its T-shaped back surface (and T-shaped front surface) is made of coplanar triangles. Consequently, there is no cycle of concave edges separating the horizontal part from the vertical part of a T-shape. This explains why the regions concerning the arms of the man placed in the lower right corner in Fig. 1 do not end at the clavicles.

A possible solution to this problem would be to add noise to the mesh. For example, the plier depicted in Fig. 10(a) essentially is a X-shaped model, which is not very well segmented because the central part of its X-shaped back surface (and Xshaped front surface) consists of coplanar triangles. By adding noise to the plier mesh (cf. Fig. 10(b)), we end up obtaining a perceptually better segmentation of the plier. But, as shown in Fig. 10(c), adding more and more noise to the mesh is not a good solution, because much likely this procedure undermines the segmentation. Possible, a better solution would be to add noise to the mesh locally (instead of globally) where needed, but this remains an open issue for future work because currently there is no way to know in advance when we need to do so.

Anyway, the important fact to retain is that the HPO segmentations agree largely with the human-generated regions of the ground truth. A number of HPO segmentations of the entire set of models (among those 380 models of the Princeton benchmark repository) are depicted in Fig. 1; also the HPO segmentations of the entire family of 20 bears is shown in Fig. 11. Moreover, the HPO algorithm compares to more recent algorithms proposed in the literature, namely those due to Au et al. [25] and Wang et al. [45]. 


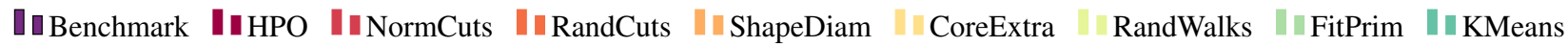

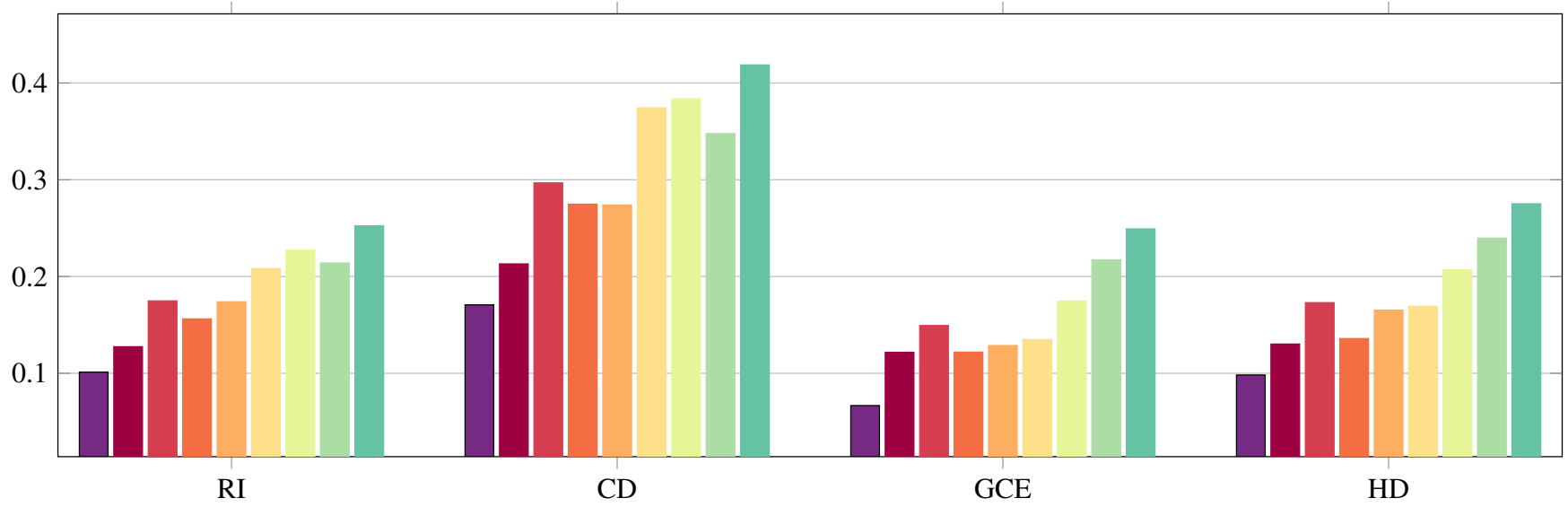

Figure 9: Comparison of segmentation algorithms with different metrics.

Table 1: Comparison of segmentation algorithms for each model category (19 categories with 20 models each).

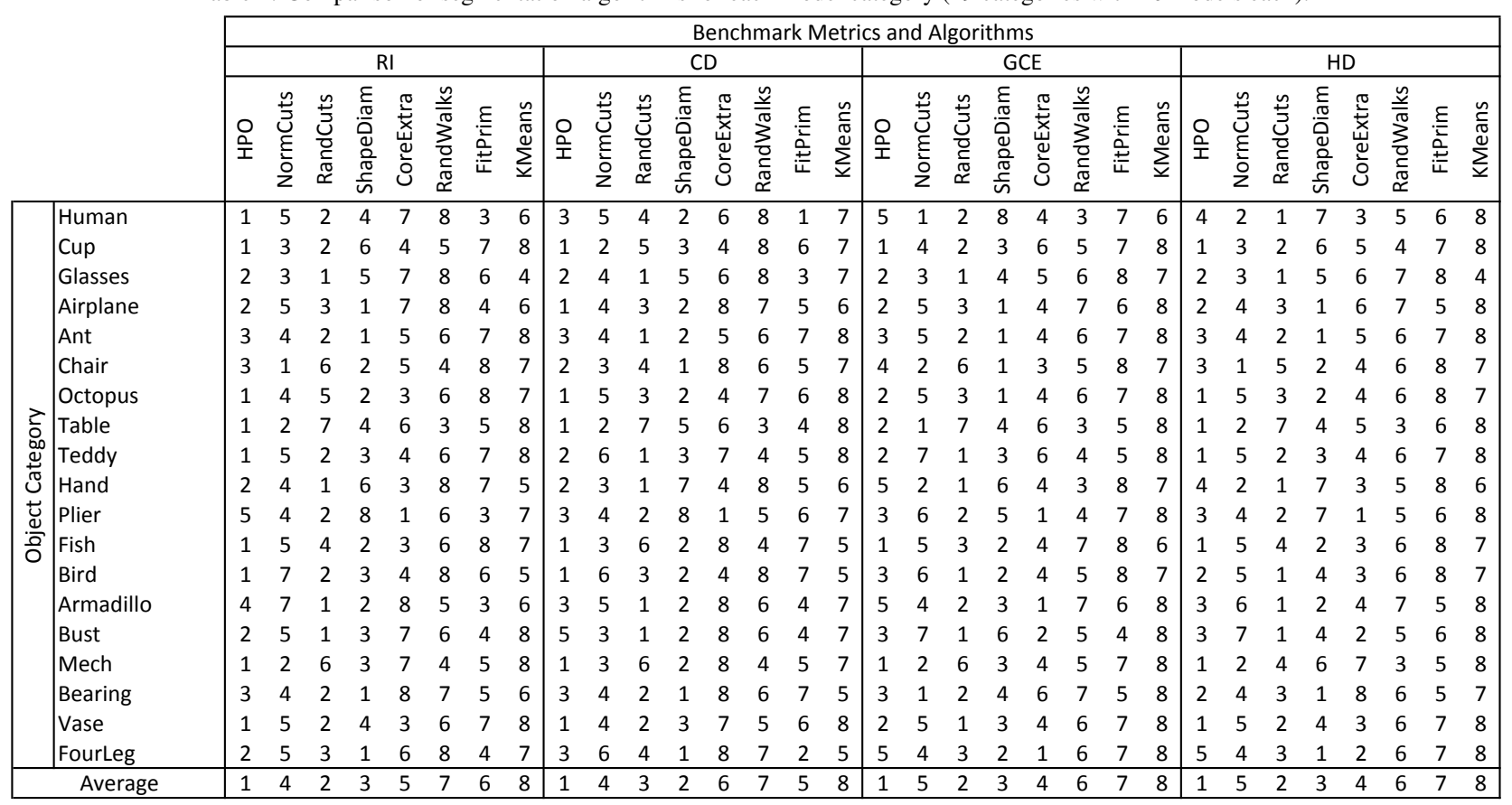

\section{Conclusions}

We have introduced here a new mesh segmentation algorithm, named HPO algorithm, that outperforms the state-of-theart algorithms in mesh segmentation in the sense that it ranks first in Princeton benchmarking for all its four metrics. This means that the HPO segmentations largely match the humangenerated segmentations of the ground truth, even using only geometric criteria (i.e., the PMT criterion, interior condition, boundary condition, and the door-in-door-out principle) and reasoning, without the need of computing convex hulls as usual in many convexity-based algorithms.

At our best knowledge, there is not any other contour-based mesh segmentation algorithm in the literature, other than the
HPO algorithm itself. The idea of a contour-based segmentation taken from image analysis and processing has led us to the concept of relaxed convexity. This in some extent avoids the over-segmentation of freeform objects. For over-segmented or noisy meshes, we have also introduced a cumulative histogram that is capable of distinguishing a noisy mesh from a mesh without significant noise, as well as a mesh smoothing filter. Furthermore, in practice, it is enough to use the region filling step to segment non-freeform objects correctly.

\section{Acknowledgements}

The authors are very grateful to reviewers for their valuable questions and suggestions that contributed to significantly 


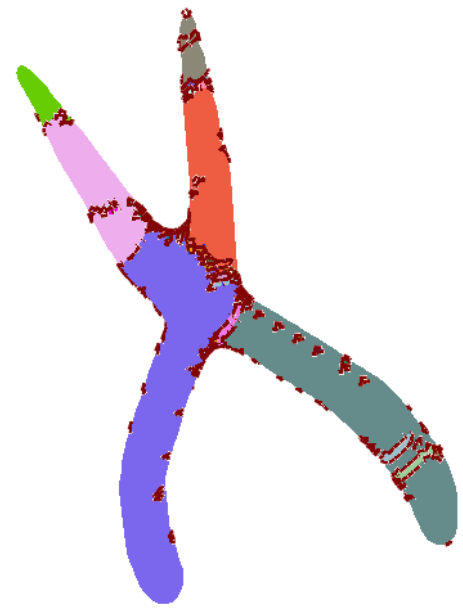

(a)

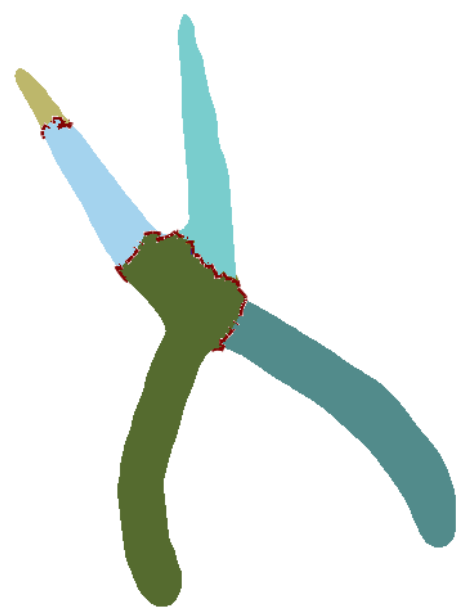

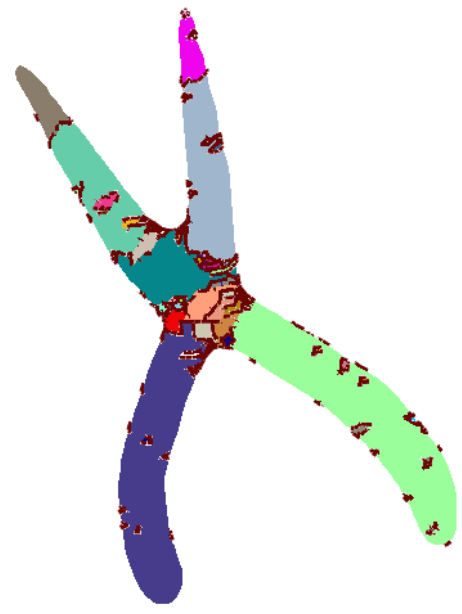

(b)

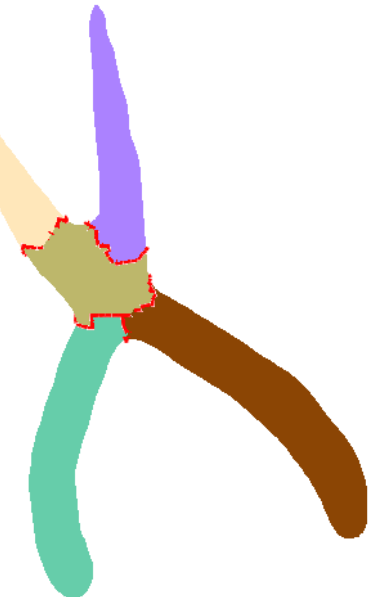

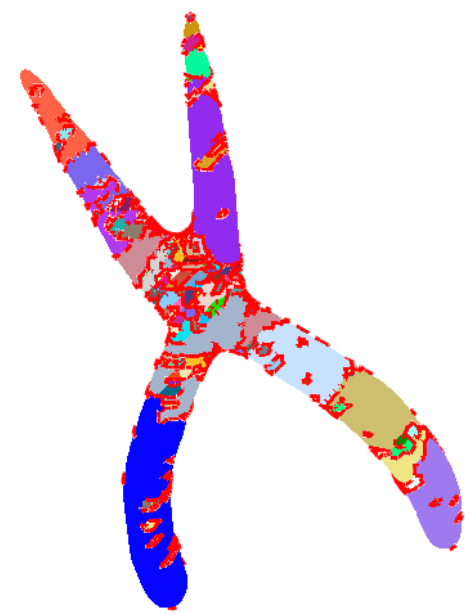

(c)

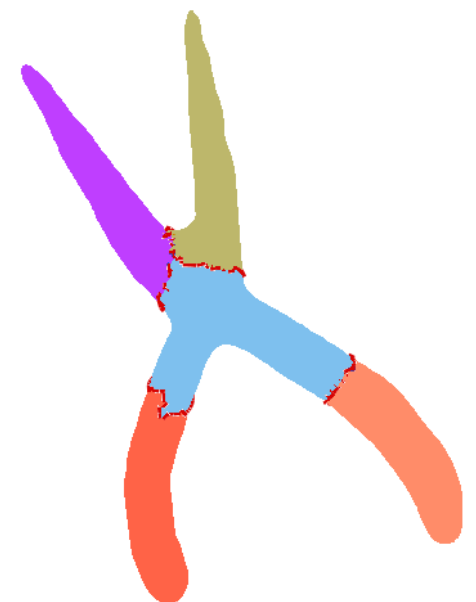

Figure 10: A plier (model 202 of Princeton benchmark) with different levels of noise, as segmented by the HPO algorithm: (a) original mesh after its filling (top) and merging (bottom) steps; (b) mesh with noise generated by vertex displacement of 0.005 , after its filling (top) and merging (bottom) steps; (c) mesh with more noise generated by vertex displacement of 0.007 , after its filling (top) and merging (bottom) steps.
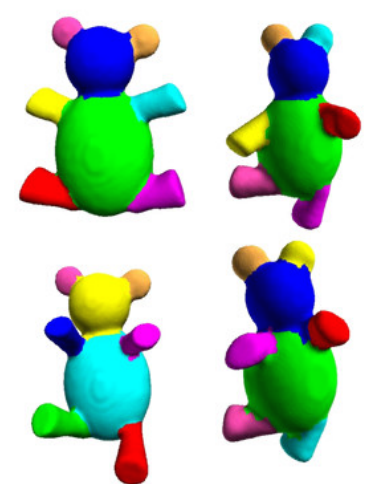
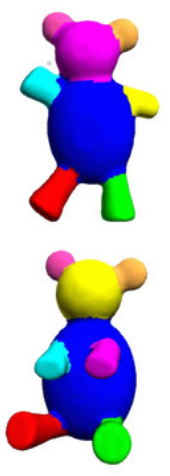
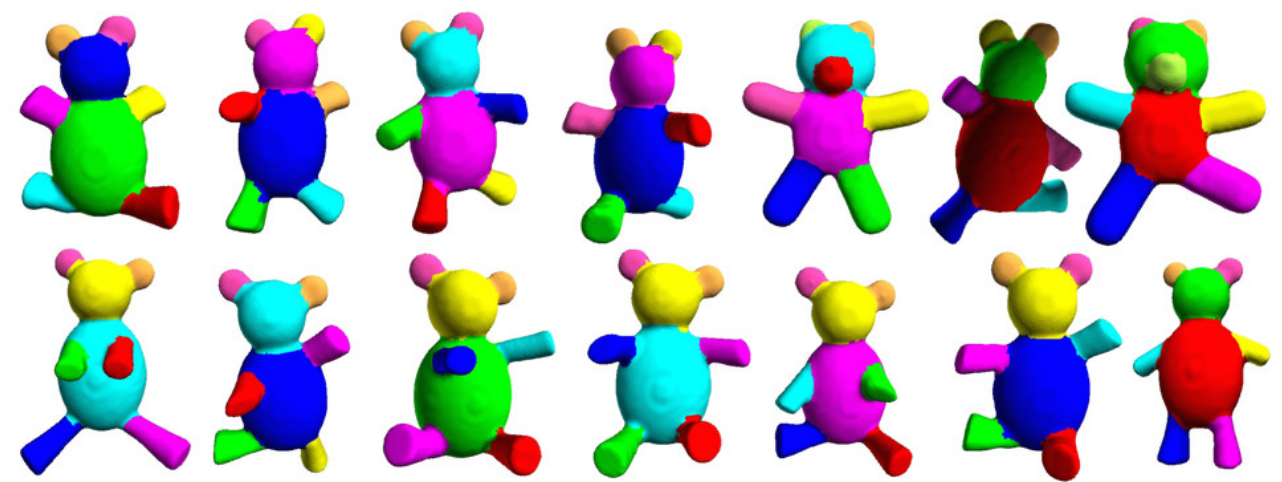

Figure 11: Models of the category of bears segmented by the HPO algorithm.

improve the article. This work was supported by FCT project UID/EEA/50008/2013.

\section{References}

[1] Eugene L. Algower and Kurt Georg. Introduction to Numerical Continuation Methods. Society for Industrial and Applied Mathematics, Philadel- 
phia, USA, 2003.

[2] Nina Amenta, Marshall Bern, and David Eppstein. Optimal point placement for mesh smoothing. Journal of Algorithms, 30(2):302-322, 1999.

[3] M. Attene, S. Katz, M. Mortara, G. Patane, M. Spagnuolo, and A. Tal. Mesh segmentation - a comparative study. In Proceedings of the IEEE International Conference on Shape Modeling and Applications (SMI'2006), pages 7-19. IEEE Computer Society, 2006.

[4] Marco Attene, Bianca Falcidieno, and Michela Spagnuolo. Hierarchical mesh segmentation based on fitting primitives. The Visual Computer, 22:181-193, 2006.

[5] C. Bajaj and T. Dey. Convex decomposition of polyhedra and robustness. SIAM Journal on Computing, 21(2):339-364, 1992.

[6] Irving Biederman. Recognition-by-components: A theory of human image understanding. Psychological Review, 94:115-147, 1987.

[7] H. Blum. A transformation for extracting new descriptors of shape. In W. Wathen-Dunn, editor, Models for the Perception of Speech and Visual Form, pages 362-380. MIT Press, 1967.

[8] Steve Capell, Seth Green, Brian Curless, Tom Duchamp, and Zoran Popovic. Interactive skeleton-driven dynamic deformations. ACM Transactions on Graphics, 21(3):586-593, 2002.

[9] B. Chazelle and L. Palios. Decomposition algorithms in geometry. In C. Bajaj, editor, Algebraic Geometry and its Applications, pages 419447. Springer-Verlag, Berlin, 1994.

[10] Bernard Chazelle, David P. Dobkin, Nadia Shouraboura, and Ayellet Tal. Strategies for polyhedral surface decomposition: an experimental study. In Proceedings of the 11th Annual Symposium on Computational Geometry, SCG '95, pages 297-305, New York, NY, USA, 1995. ACM Press.

[11] Bernard M. Chazelle. Convex decompositions of polyhedra. In Proceedings of the 13th Annual ACM Symposium on Theory of Computing, STOC '81, pages 70-79, New York, NY, USA, 1981. ACM

[12] Lijun Chen and NicolasD. Georganas. An efficient and robust algorithm for $3 \mathrm{~d}$ mesh segmentation. Multimedia Tools and Applications, 29(2):109-125, 2006

[13] Xiaobai Chen, Aleksey Golovinskiy, and Thomas Funkhouser. A benchmark for 3D mesh segmentation. ACM Transactions on Graphics, 28(3):73:1-73:12, August 2009.

[14] Michael Garland, Andrew Willmott, and Paul S. Heckbert. Hierarchical face clustering on polygonal surfaces. In Proceedings of the 2001 Symposium on Interactive 3D Graphics, I3D '01, pages 49-58, New York, NY, USA, 2001. ACM.

[15] Mukulika Ghosh, Nancy M. Amato, Yanyan Lu, and Jyh-Ming Lien. Fast approximate convex decomposition using relative concavity. ComputerAided Design, 45(2):494 - 504, 2013.

[16] Aleksey Golovinskiy and Thomas Funkhouser. Randomized cuts for 3D mesh analysis. ACM Transactions on Graphics, 27(5):145:1-145:12, December 2008.

[17] Rafael Gonzalez and Richard Woods. Digital Image Processing. Prentice Hall, Inc., New Jersey, USA, 2008.

[18] Leonard R. Herrmann. Laplacian-isoparametric grid generation scheme Journal of the Engineering Mechanics Division, 102(5):749-756, 1976.

[19] Tan-Chi Ho and Jung-Hong Chuang. Volume based mesh segmentation. Journal of Information Science and Engineering, 28(4):705-722, July 2012.

[20] Donald D. Hoffman and Manish Singh. Salience of visual parts. Cognition, 63(1):29 - 78, 1997.

[21] Alan D. Kalvin and Russell H. Taylor. Superfaces: Polygonal mesh simplification with bounded error. IEEE Computer Graphics $\mathcal{E}$ Applications, 16:64-77, May 1996.

[22] Sagi Katz, George Leifman, and Ayellet Tal. Mesh segmentation using feature point and core extraction. The Visual Computer, 21:649-658, 2005.

[23] Sagi Katz and Ayellet Tal. Hierarchical mesh decomposition using fuzzy clustering and cuts. ACM Transactions on Graphics, 22:954-961, July 2003.

[24] Hyoungseok B. Kim and Hosook Kim. Mesh segmentation based on local geometric properties. International Journal of Computer Science and Network Security, 10(1), 2010.

[25] Oscar Kin-Chung Au, Youyi Zheng, Menglin Chen, Pengfei Xu, and Chiew-Lan Tai. Mesh segmentation with concavity-aware fields. IEEE Transactions on Visualization and Computer Graphics, 18(7):1125-1134, July 2012.
[26] V. Kraevoy, D. Julius, and A. Sheffer. Shuffler: Modeling with interchangeable parts. Technical report, TR-2006-09, Department of Computer Science, University of British Columbia, 2006.

[27] Sheffer A. Kraevoy V, Julius D. Shuffler: modeling with interchangeable parts. The Visual Computer, 2007., 2007.

[28] Yu-Kun Lai, Shi-Min Hu, Ralph R. Martin, and Paul L. Rosin. Fast mesh segmentation using random walks. In Proceedings of the 2008 ACM Symposium on Solid and Physical Modeling, SPM '08, pages 183-191, New York, NY, USA, 2008. ACM.

[29] Jyh-Ming Lien and Nancy M. Amato. Approximate convex decomposition of polyhedra. In ACM SIGGRAPH 2004 Posters, SIGGRAPH '04, New York, NY, USA, 2004. ACM.

[30] Jyh-Ming Lien and Nancy M. Amato. Approximate convex decomposition of polygons. Computational Geometry: Theory and Applications, 35:100-123, August 2006.

[31] Hairong Liu, Wenyu Liu, and L.J. Latecki. Convex shape decomposition. In Computer Vision and Pattern Recognition (CVPR), 2010 IEEE Conference on, pages $97-104$, june 2010.

[32] Rong Liu and Hao Zhang. Mesh segmentation via spectral embedding and contour analysis. Computer Graphics Forum, 26(3):385-394, 2007.

[33] Alan Mangan and Ross Whitaker. Partitioning 3D surface meshes using watershed segmentation. IEEE Transactions on Visualization and Computer Graphics, 5(4):308-321, October 1999.

[34] Joshua Podolak, Philip Shilane, Aleksey Golovinskiy, Szymon Rusinkiewicz, and Thomas Funkhouser. A planar-reflective symmetry transform for 3d shapes. ACM Transactions on Graphics, 25:549-559, July 2006.

[35] Martin Reuter, Silvia Biasotti, Daniela Giorgi, Giuseppe Patanè, and Michela Spagnuolo. Discrete laplace-beltrami operators for shape analysis and segmentation. Computers and Graphics, 33:381-390, June 2009.

[36] P. Sander, Z. Wood, S. Gortler, J. Snyder, and H. Hoppe. Multi-chart geometry images. In Proceedings of the 2003 Eurographics/ACM SIGGRAPH Symposium on Geometry Processing, pages 146-155. Eurographics Association, 2003.

[37] Ariel Shamir. A survey on mesh segmentation techniques. Computer Graphics Forum, 27(6):1539-1556, September 2008.

[38] L. Shapira, A. Shamir, and D. Cohen-Or. Consistent mesh partitioning and skeletonisation using the shape diameter function. The Visual Computer, 24(4):249-259, 2008.

[39] Y. Shinagawa, T. L. Kunii, and Y. L. Kergosien. Surface coding based on morse theory. IEEE Computer Graphics and Applications, 11(5):66-78, 2001.

[40] Shymon Shlafman, Ayellet Tal, and Sagi Katz. Metamorphosis of polyhedral surfaces using decomposition. Computer Graphics Forum, 21(3):219-228, 2002.

[41] Kaleem Siddiqi, Ali Shokoufandeh, Sven J. Dickinson, and Steven W. Zucker. Shock graphs and shape matching. In Proceedings of the 6th IEEE International Conference on Computer Vision, pages 222-229, Bombay, India, 1998. IEEE Press.

[42] J. Tierny, J.-P. Vandeborre, and M. Daoudi. Topology driven 3d mesh hierarchical segmentation. In Proceedings of the IEEE International Conference on Shape Modeling and Applications, pages 215-220. IEEE Press, 2007.

[43] R.B. Tilove. Set membership classification: a unified approach to geometric intersection problems. IEEE Transactions on Computers, C29(10):874-883, October 1980.

[44] J. Vollmer, R. Mencl, and H. Mller. Improved laplacian smoothing of noisy surface meshes. Computer Graphics Forum, 18(3):131-138, 1999.

[45] Hao Wang, Tong Lu, Oscar Kin-Chung Aub, and Chiew-Lan Tai. Spectral $3 \mathrm{~d}$ mesh segmentation with a novel single segmentation field. Graphical Models, 76(5):440-456, 2014.

[46] Eugene Zhang, Konstantin Mischaikow, and Greg Turk. Feature-based surface parameterization and texture mapping. ACM Transactions on Graphics, 24:1-27, 2005.

[47] H. Zhang, O. van Kaick, and R. Dyer. Spectral mesh processing. Computer Graphics Forum, 29(6):1865-1894, 2010.

[48] Emanoil Zuckerberger, Ayellet Tal, and Shymon Shlafman. Polyhedral surface decomposition with applications. Computers $\mathcal{E}$ Graphics, 26(5):733-743, 2002. 

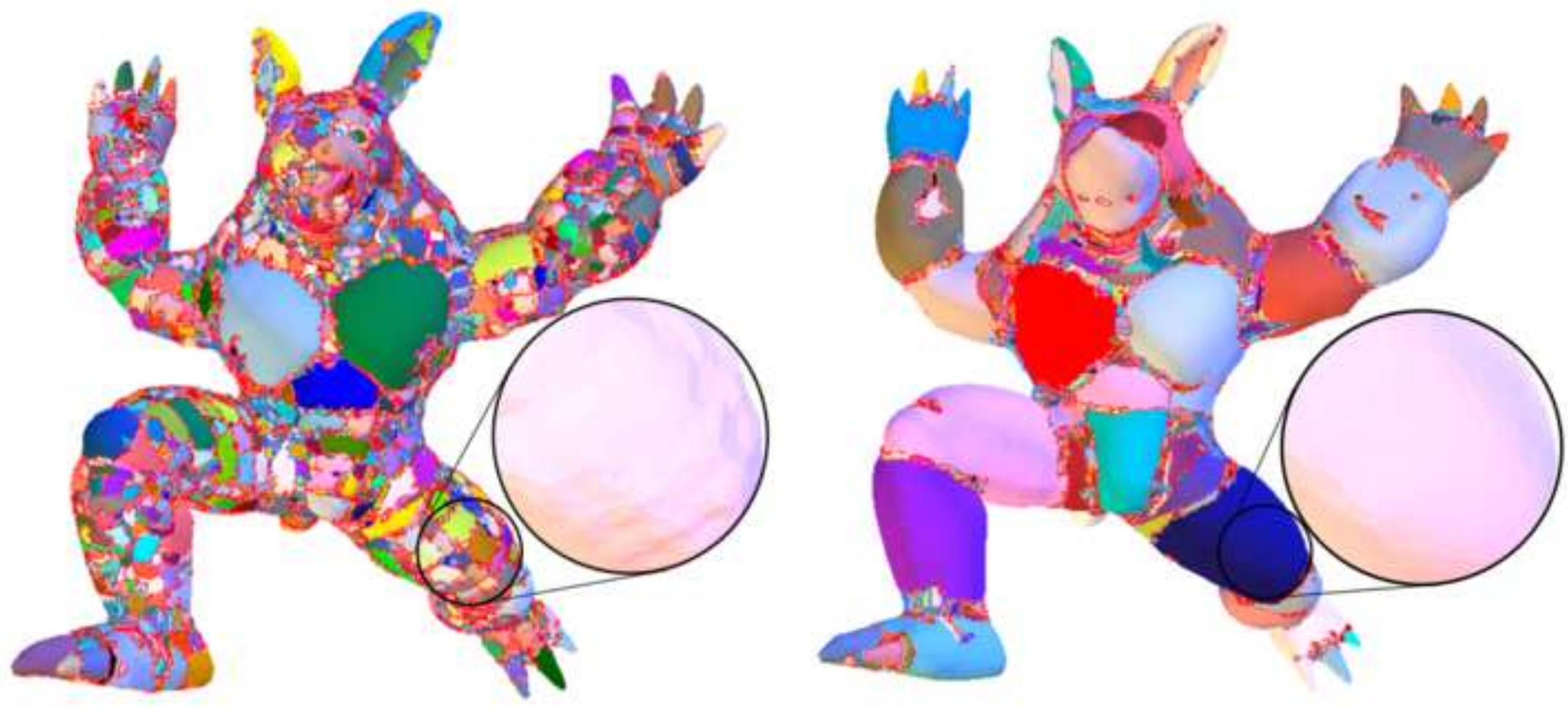